\title{
Target identification and lead discovery by functional lipidomics
}

\author{
"...methodological and technical advances in chromatography and \\ mass spectrometry allow for the first time comprehensive insights into \\ lipid diversity."
}

First draft submitted: 14 September 2016; Accepted for publication: 19 September 2016; Published online: 15 November 2016

Keywords: bioactive lipid $\bullet$ cancer $\bullet$ cell signaling $\bullet$ compound screening $\bullet$ fatty acid - inflammation $\bullet$ lipidomics $\bullet$ mass spectrometry $\bullet$ phospholipids $\bullet$ protein-lipid interaction

Lipids are structurally highly diverse biomolecules that form membranes and contribute to essentially all cellular processes. How they affect protein structure and function is widely unknown and systematic approaches to identify bioactive lipid mediators that specifically interact with proteins are only emerging [1]. Specific protein-lipid interactions are not restricted to distinct lipid classes, such as eicosanoids, lysophospholipids or phosphoinositides, which are generated from structural lipids by enzymatic conversion [2]. Recent studies suggest that a broad range of lipids, including phospholipids, specifically interact with proteins and influence their structure and function [2]. In light of the enormous diversity of lipids with thousands of different species in each cell, it is very likely that a large set of protein-lipid interactions exist with unexplored physiological relevance and pharmacological potential.

Recent advances in chromatography and mass spectrometry allow for the first time comprehensive insights into complex cellular lipid networks [3]. Large efforts have been undertaken to monitor the lipid composition in health and disease [4]. An increasing number of system biological studies correlates lipidomic data with gene expression, metabolic profiles, cell signaling and phenotypes, thereby focusing on the discovery of biomarkers and lipid signatures [5]. Less understood is the role of individual lipid ligands as well as their biosynthesis and interaction with target proteins - knowledge that is essential for the structure-based design of both lipid mimetics and small molecules that selectively modulate the cellular lipid composition [5].

'Functional lipidomics' represents an integrative strategy for the identification of bioactive lipids, their molecular targets as well as drug candidates that selectively modulate the cellular lipid composition. Target identification by functional lipidomics combines comprehensive lipid analytics with a broad spectrum of chemical biological, biochemical, cell biological and molecular pharmacological tools. In a first step, lipids are profiled in relevant (disease) models and compared with (healthy) controls. Lipokine-like functions are expected among those lipids that are organized in co-regulated lipid clusters and are exceptionally regulated. Correlation of the relative lipid abundance with biological readouts, for example, the regulation of signaling pathways, provides initial insights into the function of bioactive lipid candidates, in particular, when referring to well-characterized model systems. In a second step, lipidinduced signaling pathways and molecular targets are characterized, which can be a challenging task because the majority of cellular lipid species is commercially not available, and indirect approaches targeting lipid metabolic isoenzymes are often hampered by the intensive co- and counter-regulations within lipid networks [6], making effects of

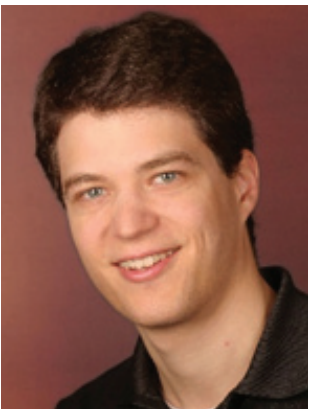

Andreas Koeberle Chair of Pharmaceutical/Medicinal Chemistry, Institute of Pharmacy, Friedrich Schiller University, Philosophenweg 14, 07743 Jena, Germany

Tel.: +493641949815 Fax: +49 3641949802 andreas.koeberle@uni-jena.de 
metabolic perturbations on the lipidome difficult to predict. From the functional profile of the potential lipid mediator, direct molecular targets are predicted and analyzed for biophysical interactions with the lipid. The latter benefits from novel in vitro and in vivo technologies for the analysis of protein-lipid complexes, including native protein mass spectrometry, lipid overlay and pull-down assays, liposome microarray-based screening systems and affinity-purification lipidomics [1].

\section{“...lipidomic profiling led to the discovery of oxylipins (including specialized proresolving lipid mediators) that either induce or resolve inflammation during influenza."}

Lead discovery and subsequent drug development may either focus on stable lipid mimetics (which often suffer from high lipophilicity, metabolic instability and plasma protein binding) or on selective ligands targeting a key enzyme within the biosynthesis or degradation of the lipid factor (which requires the knowledge of the metabolic pathway that might differ between cell types and experimental conditions) [5]. Lipidomics-based phenotypic screening represents an alternative: a representative pattern of the lipidome is addressed, instead of focusing on defined molecular targets. This approach allows for simultaneous screening of small molecules with different mode of action and concomitantly provides comprehensive insights into the lipid network to estimate their selectivity and safety. Still disadvantageous is the poor compatibility to high-throughput screening, due to limitations in automatic data analysis rather than instrumental performance. In fact, ultraperformance LC and high sensitivity ESI-tandem mass spectrometry meanwhile allow the simultaneous analysis of hundreds of lipids within a couple of minutes [7].

Several recent studies successfully implemented the concept of functional lipidomics and discovered novel lipid mediators with promising pharmacological potential aside of established bioactive lipid classes [3-5]. For example, Yore $e t$ al. used a quantitative mass spectrometry platform together with accurate mass measurements to identify branched fatty acid esters of hydroxyl fatty acids as Glut4-regulated lipids in murine adipose tissue [8]. Further biological evaluation revealed that these endogenous lipid mediators are regulated by dietary fat intake, activate the fatty acid membrane receptor GPR120 and induce insulin secretion and sensitivity with potential impact for the treatment of Type 2 diabetes. Accordingly, lipidomic profiling led to the discovery of oxylipins (including specialized proresolving lipid mediators) that either induce or resolve inflammation during influenza [9], bacterial infec- tion [10], tissue regeneration [11] and diverse inflammatory diseases [12]. Of particular interest is also the combination of lipidomics with other Omics technologies, in particular transcriptomics, (phospho)proteomics and epigenomics to correlate changes in metabolism to the expression and activation of proteins. For example, an integrated metabolic and proteomic mapping platform together with activity-based protein profiling revealed that PAF acetylhydrolase $1 \mathrm{~B} 3$ promotes breast cancer progression [13].

All examples so far refer to lipid mediators whose lipokine-like functions depend on their unique structure, which is accomplished by conjugating or oxidizing enzymes. However, there is increasing evidence that also nonmodified lipids, such as membrane phospholipids, specifically interact with proteins and selectively induce signaling pathways and cellular responses [14-17]. Functional lipidomics led to the discovery of unexpected bioactive lipids including phosphatidylcholines containing polyunsaturated fatty acid [17] and distinct phosphatidylinositols [18,19]. Phosphatidylcholines containing polyunsaturated fatty acid, for example, oscillate during the cell cycle and suppress cell proliferation by reducing the affinity of the serine-threonine kinase Akt to phosphatidylinositol-3,4,5-trisphosphates that act as membrane anchor of proteins with pleckstrin homology domain [17].

In conclusion, methodological and technical advances in chromatography and mass spectrometry allow for the first time comprehensive insights into lipid diversity [3]. This progress is accompanied by a fundamental change in our understanding of how lipids regulate protein function. The majority of cellular lipids have long been considered to possess mainly structural, storage and energy functions, except for small lipid subgroups, such as eicosanoids, whose importance for inflammatory and immune-modulatory signal transduction has been known since decades [20]. While the biological relevance of lipids is unquestioned, we only begin to understand the pleiotropic relations between lipid diversity and protein function. Many questions remain open, despite the increasing number of structurally diverse lipid mediators reported from lipidomics studies. How are the proposed lipid mediators biosynthesized, and how are they regulated? What are their molecular targets, are they selectively affected and is their modulation relevant for disease? Taken together, our current knowledge about complex lipid-protein regulatory networks is still fragmentary. However, a detailed understanding of the interacting network, as accessible by functional lipidomics, is required to systematically relate potential lipid mediators to target proteins and functions and to open the door to the widely unexplored universe of protein-lipid interactions for drug discovery. Moreover, functional lipidomics might help to predict pharmacological activities and 
side effects of small molecules by phenotypic lipidomic screening, thereby improving candidate selection during an early stage of drug development.

\section{Financial \& competing interests disclosure}

The author has no relevant affiliations or financial involvement with any organization or entity with a financial interest

\section{References}

1 Saliba AE, Vonkova I, Gavin AC. The systematic analysis of protein-lipid interactions comes of age. Nat. Rev. Mol. Cell Biol. 16(12), 753-761 (2015).

2 Kimura T, Jennings W, Epand RM. Roles of specific lipid species in the cell and their molecular mechanism. Prog. Lipid Res. 62, 75-92 (2016).

3 Han X. Lipidomics for studying metabolism. Nat. Rev. Endocrinol. doi:10.1038/nrendo.2016.98 (2016) (Epub ahead of print).

4 Yang L, Li M, Shan Y, Shen S, Bai Y, Liu H. Recent advances in lipidomics for disease research. J. Sep. Sci. 39(1), 38-50 (2016).

5 Vihervaara T, Suoniemi M, Laaksonen R. Lipidomics in drug discovery. Drug Discov. Today 19(2), 164-170 (2014).

6 Hermansson M, Hokynar K, Somerharju P. Mechanisms of glycerophospholipid homeostasis in mammalian cells. Prog. Lipid Res. 50(3), 240-257 (2011).

7 Brugger B. Lipidomics: analysis of the lipid composition of cells and subcellular organelles by electrospray ionization mass spectrometry. Annu. Rev. Biochem. 83, 79-98 (2014).

8 Yore MM, Syed I, Moraes-Vieira PM et al. Discovery of a class of endogenous mammalian lipids with anti-diabetic and anti-inflammatory effects. Cell 159(2), 318-332 (2014).

9 Tam VC. Lipidomic profiling of bioactive lipids by mass spectrometry during microbial infections. Semin. Immunol. 25(3), 240-248 (2013).

10 Dalli J, Chiang N, Serhan CN. Elucidation of novel 13-series resolvins that increase with atorvastatin and clear infections. Nat. Med. 21(9), 1071-1075 (2015).

11 Dalli J, Sanger JM, Rodriguez AR, Chiang N, Spur BW, Serhan CN. Identification and actions of a novel third in or financial conflict with the subject matter or materials discussed in the manuscript. This includes employment, consultancies, honoraria, stock ownership or options, expert testimony, grants or patents received or pending, or royalties.

No writing assistance was utilized in the production of this manuscript.

maresin conjugate in tissue regeneration: MCTR3. PLoS ONE 11(2), e0149319 (2016).

12 Dennis EA. Lipid cell signaling, enzymes, LIPID MAPS, and mediators of inflammation. J. Biol. Chem. doi:10.1074/ jbc.X116.723791 (2016) (Epub ahead of print).

13 Mulvihill MM, Benjamin DI, Ji X et al. Metabolic profiling reveals PAFAH1B3 as a critical driver of breast cancer pathogenicity. Chem. Biol. 21(7), 831-840 (2014).

14 Laganowsky A, Reading E, Allison TM et al. Membrane proteins bind lipids selectively to modulate their structure and function. Nature 510(7503), 172-175 (2014).

15 Contreras FX, Ernst AM, Haberkant P et al. Molecular recognition of a single sphingolipid species by a protein's transmembrane domain. Nature 481(7382), 525-529 (2012).

16 Maeda K, Anand K, Chiapparino A et al. Interactome map uncovers phosphatidylserine transport by oxysterol-binding proteins. Nature 501(7466), 257-261 (2013).

17 Koeberle A, Shindou H, Koeberle SC, Laufer SA, Shimizu T, Werz O. Arachidonoyl-phosphatidylcholine oscillates during the cell cycle and counteracts proliferation by suppressing Akt membrane binding. Proc. Natl Acad. Sci. USA 110(7), 2546-2551 (2013).

18 Gil-De-Gomez L, Astudillo AM, Meana C et al. A phosphatidylinositol species acutely generated by activated macrophages regulates innate immune responses. J. Immunol. 190(10), 5169-5177 (2013).

19 Koeberle A, Pergola C, Shindou H et al. Role of p38 mitogen-activated protein kinase in linking stearoylCoA desaturase-1 activity with endoplasmic reticulum homeostasis. FASEB J. 29 (6), 2439-2449 (2015).

20 Dennis EA, Norris PC. Eicosanoid storm in infection and inflammation. Nat. Rev. Immunol. 15(8), 511-523 (2015). 